\title{
Case-based reasoning adaptation of numerical representations of human organs by interpolation
}

\author{
Julien Henriet(1), Pierre-Emmanuel Leni(1), Rémy Laurent(1), Michel \\ $\operatorname{Salomon}(2)$ \\ (1) Université de Franche-Comté, Chrono-Environnement UMR 6249 CNRS \\ 4 Place Tharradin, 25200 Montbéliard, France \\ (2) Université de Franche-Comté, FEMTO-ST UMR 6174 CNRS \\ Rue Engel Gros, 90000 Belfort, France \\ julien.henriet,pierre-emmanuel.leni,remy.laurent,michel.salomon@univ-fcomte.fr
}

\begin{abstract}
Case-Based Reasoning (CBR) and interpolation tools are capable of providing solutions to unknown problems through the adaptation of other problems already solved. This paper proposes a generic approach using interpolation tool during the CBR-adaptation phase. Modelling was applied to EquiVox which attempts to design three dimensional representations of human organs according to external measurements. EquiVox follows the CBR-cycle and its adaptation tool is based on Artificial Neural Networks. The performances of this adaptation tool are evaluated and discussed. The results show that EquiVox fulfills the requirements of radiation protection experts who use such prototypes while also showing the limits of use of interpolation tools in CBR-adaptation. When adaptations are guided by experience and by the trial and error of experts, interpolation tools are well-fitted methods to automatically and quickly provide adaptation strategies and knowledge through training phases.
\end{abstract}

Keywords: Case-based reasoning, adaptation, interpolation, artificial neural network, radiation protection, 3D numerical phantoms.

\section{Introduction}

Case-Based Reasoning (CBR) is a problem solving method that uses similar solutions from similar past problems in order to solve new prob-

lems [1]. One of the main properties of the CBR system is its ability to 
adapt known solutions to unknown ones. Many adaptation strategies can be found in the literature. Adaptation by generalisation/specialisation requires a hierarchical organisation of the CBR source cases according to generalisation/specialisation relations. Some characteristics are hidden in the generalisation process whereas special ones are added to the general case during the specialisation process.

Adaptation through the use of rules [2] consists of computing the solution of a target case by applying a function to it, using as parameters the solution to a source case that presents some similarities.

Differential adaptation [3] is based on the evaluation of variations found between source and target cases: an approximate solution of the target case is computed by applying the variations between the target case and the source case to the solution of the source case under consideration.

Conservative adaptation [4] is based on Revision Theory that considers knowledge modifications. This kind of adaptation is based on minimising modifications applied to knowledge. A cost for the possible adaptations must be computed.

Some studies have highlighted the advantages of prototype-based classifications for CBR-systems [5, 6, 7]. These approaches consist of choosing prototypes as representatives for each class of case. Prototypes are also interesting when solutions are not well-known. For instance, considering someone in front of you, even if it is not possible to model an accurate three-dimensional (3D) representation of his/her lung contours without 3D medical scans, it is possible to create a prototype of these organ contours. In such a case, interpolation tools may provide prototypes of an accurate representation of target cases.

Furthermore, when rules can be discovered only through experience, interpolation tools become the method best suited to adapting solution(s) of source case(s) to target case(s). In addition, interpolation tools can be trained and automatically provide the required adaptation knowledge. The drawback of such a method is that it is not intelligible to users since the interpolation tool functions as a black box [8]. B. Pandey and R.B. Michra proposed a CBR-based systems that uses Artificial Neural Network (ANN) but during the retrieval phase [9].

In the first part of this paper we have modelled how interpolation tools can be used during the adaptation phase of CBR-based systems. In the second part we present and analyse the example of the EquiVox application in which ANNs were implemented in order to adapt 3D numerical representations of 
organ contours. The results obtained are then presented and discussed.

\section{Method}

\subsection{Case model}

In CBR approaches, a case is defined as a $\{$ problem, solution $\}$ association. The problem part can be represented using a set of $n$ descriptors and the solution by means of a set of $N$ elements. Thus, a source case $s$ is defined as follows:

$s=\left\{p b^{s}, s o l^{s}\right\}=\left\{\left\{d_{i}^{s}\right\}_{i \in\{1, \ldots, n\}},\left\{e_{i}^{s}\right\}_{i \in\{1, \ldots, N\}}\right\}$

and a target case $t$ as:

$t=\left\{p b^{t}\right.$, sol $\left.^{t}\right\}=\left\{\left\{d_{i}^{t}\right\}_{i \in\{1, \ldots, n\}},\left\{e_{i}^{t}\right\}_{i \in\{1, \ldots, N\}}\right\}$

\subsection{Retrieval phase}

The retrieval phase lies in sorting the source cases according to their similarity with the target case. We implemented a classical version of the $k$-Nearer Neighbour (kNN) algorithm [10] which computes the distances between each descriptor of the problem parts of target and source cases.

Thus, for each source case $i$, a similarity index $S_{i}$ is computed as follows:

$$
S_{i}=\frac{\sum_{k=1}^{n} \frac{\Delta_{k}-\left|d_{k}^{i}-d_{k}^{t}\right|}{\Delta_{k}}}{n}
$$

where $\Delta_{k}$ is the difference between the maximum and the minimum known values that the descriptor $d_{k}$ can take. The $S_{i}$ value is always between 0 and 1. The greater the similarity of $i$ to $t$, the closer the $S_{i}$ value to 1 .

\subsection{Adaptation phase}

In [11] B. Knight and F.L. Woon used the interpolation method of D. Shepard [12] to retrieve and adapt a solution to a target case over nominal values. Indeed, they have a limited number of possible solutions and the adapted solution $s o l^{t}$ of $t=\left\{p b^{t}\right.$, sol $\left.^{t}\right\}$ minimizes $f\left(s^{t} l^{t}\right)=\frac{\sum_{i=1}^{2}\left(\operatorname{dist}_{s o l}\left(\operatorname{sol}^{t}, s^{i}\right)^{2} . \operatorname{dist}_{p b}\left(p b^{t}, p b^{i}\right)^{-1}\right)}{\sum i=1^{2}\left(\operatorname{dist}_{p b}\left(p b^{t}, p b^{i}\right)^{-1}\right)}$ where dist $_{p b}$ (respectively dist $_{s o l}$ ) is the distance in the problem (respectively solution) space and where cases $i=1$ and $i=2$ are the source cases most similar to $t$ for which $p b^{1} \leq p b^{t} \leq p b^{2}$.

In [13] A. Cordier et al. used Influence functions that link variations in problem descriptors to those in solution descriptors. They also used 
Dependencies that are relationships between problem and solution descriptors. These Dependencies indicate whether each problem descriptor impacts each solution element (Dependency $\left(e_{i}, d_{j}\right)=T R U E$ if $e_{i}$ depends on $d_{j}$, Dependency $\left(e_{i}, d_{j}\right)=F A L S E$ otherwise). In fact, the Influence function of $e_{i}$ takes as parameters the set of $d_{j}$ for which Dependency $\left(e_{i}, d_{j}\right)=T R U E$.

Our approach merges these two, considering interpolation tools as influence functions of $e_{i}^{t}, \forall i \in\{1, \ldots, N\}$. Thus, we assume $S=\left\{p b^{S}, s^{S} l^{S}\right\}$ is the source case most similar to $t$, which means $S_{S} \leq S_{i} \forall i \in \Omega$ where $\Omega$ is the set of all the source cases. For each $e_{i}, i \in\{1, \ldots, N\}$, we have considered the entire set of problem descriptor items $\Omega_{d e p}^{i}=\cup\{j\}$ for which Dependency $\left(e_{i}, d_{j}\right)=T R U E$. The interpolation function Interpolate $_{i}()$ of $e_{i}$ used as Influence function is then given by Equation 2:

$$
e_{i}^{t}=\text { Interpolate }_{i}\left(\cup_{j \in \Omega_{d e p}^{i}}\left\{d_{j}^{t}-d_{j}^{S}\right\}, \cup_{j \in \Omega_{d e p}^{i}}\left\{d_{j}^{S}\right\}, e_{i}^{S}\right)
$$

\section{EquiVox application}

In case of accidental exposure to radiation, a dosimetry evaluation must be established for each potential victim (subject) as soon as possible. Most of the time, this evaluation is based on available $3 D$ voxel phantoms, numerical models created from medical images to represent a subject with maximum realism. Examples of voxel phantoms for dosimetric assessment following internal contamination or external exposure can be found in $[14,15]$. However, even when medical images are available, the subject's specific phantom is not always accessible since its construction is time consuming and requires expert knowledge. Moreover, medical images are avoided so as to prevent any additional exposure to radiation. Thus, existing models are used even if their characteristics differ from the subject's biometrical data. Nevertheless, dosimetry assessment accuracy and the resulting decontaminating medical action are highly dependent on the similarity between phantom and subject. Hence, the actual work aims at assisting the physician in choosing and customising the most similar phantom from the existing and available ones.

A large number of $C B R$ designed for Health Science (CBR-HS) can be found in $[16,17]$. Combinations with Artificial Intelligence (AI) tools can also be found in $[18,19,20]$. In [21] E.B. Reategui et al. combined neural networks with CBR in a diagnostic system for congenital heart diseases. In their approach, the neural network is trained with the cases stored in the library, and is used during the consultation process to make hypotheses of possible 
diagnostic solutions and to guide the search for similar cases. EquiVox [22] is a CBR-HS system that also combines an AI tool to a CBR-HS system, but during the adaptation process instead of the retrieving process like in E.B. Reategui's approach. Indeed, EquiVox uses the CBR-approach to find the most similar phantom(s) within any set of phantoms and then attempts to adapt them to the characteristics of the target case (the subject) using Artificial Neural Networks (ANNs) [23]. At this step of our work, EquiVox processes 3D contours of three organs: lungs, heart and oesophagus.

A large number of phantoms can be found in the literature [24, 25], and radiation protection is also divided into numerous sub-domains. Indeed, some phantoms are commonly used by experts for external radiotherapy, and others are used by other physicians for evaluation of internal doses received. In fact, each expert has their own collection of 10 to 20 phantoms. When physician's usual phantoms are all too distant from the subject, the expert must create a new one. Practically, using iterative 3D dilations and contractions, physicians modify the contours of the 3D organs of their phantoms until they correspond to those of the subject. They then assemble them to obtain the final phantom on which the computations will be based [25]. In addition, these transformations are only driven by experience and trials and errors, and may take many hours or more. The delay also increases with the number of subjects, whereas the problem resolution delay may be limited. Indeed, in the case of massive irradiation for example, when a disaster such as a nuclear explosion occurs, dosimetric reports are required for hundreds of people of different sizes. In fact, the creation of new organ contours requires a fast data-driven method, and since there is no physical law to govern its design, the experts are not able to explicit a rule for the transformation of an organ contour. Thus, the main challenge for EquiVox is to reproduce the same transformation process automatically, without human intervention. Another requirement is that every subject increases the accuracy of dose calculations. Current implementation relies on phantoms usually used by a team of experts for pulmonary anthroporadiametry which consists of evaluating the internal dose inhaled [26].

Figure 1 presents the technologies that were used and the data flows over the EquiVox architecture. The whole phantoms are all stored under Rhino3D files [27]. The corresponding biometric data are stored under an ontology scheme (data flow $\sharp 0$ ). The contours of the lungs, heart and oesophagus are extracted (data flow $\sharp 1$ ) and then transmitted to the ANN training module (data flow $\sharp 2$ ), which creates three ANNs (data flow $\sharp 3$ ): one per organ. 
When a new phantom is required, the target case description is transmitted to the retrieval module (data flow $\sharp 4$ ) which determines the similitude index by taking into account the source cases (data flow \#5). If required by the experts, the thorax adaptation module sends the characteristics of the source cases (data flow $\sharp 6$ ) to the ANN interpolation module (data flow $\sharp 7$ ) which loads the trained ANNs (data flow $\sharp 8$ ) and the coordinates of the contour of the organ in question (data flow $\sharp 9$ ) in order to create interpolated contours suited to the target case (data flow $\sharp 10$ ), which are combined to create the interpolated organ contours (data flow $\sharp 11$ ).

\subsection{Case modeling}

When radiation overexposure occurs, a dosimetric report must be established for all subjects. For every subject, the expert's first task is to choose the most accurate 3D phantom considering the subject's height. Indeed, a study by I. Clairand et al. demonstrated that the volume and shape of the lungs depend only on the subject's height in [28]. In addition, heart and oesophagus occupy the empty areas between the lungs. Thus, in EquiVox, a problem part of a case $i$ is described with the subject's height $p b^{i}=\left\{h_{i}\right\}$. The solution part is a set of $33 \mathrm{D}$ contours of organs: sol $^{i}=\left\{P_{i}\right\}=$ $\left\{P_{\text {Lungs }}^{i}, P_{\text {Heart }}^{i}, P_{\text {Oesophagus }}^{i}\right\}$ where $\forall O \in\{$ Lungs, Heart, Oesophagus $\}, P_{O}^{i}$ is a set of $q_{O}$ points joined by a Delauney mesh [29]: $P_{O}^{i}=\left\{C_{1}^{i, O}, \cdots, C_{q}^{i, O}\right\}$ where $C_{j}^{i, O}$ denotes the $3 \mathrm{D}$ coordinates of the point $j$ of the organ $O$ of the case $i$. Finally, a case $i$ is equal to $\left\{h_{i},\left\{P_{\text {Lungs }}^{i}, P_{\text {Heart }}^{i}, P_{\text {Oesophagus }}^{i}\right\}\right.$. As before, $t$ remains the target case.

\subsection{Retrieval phase}

The purpose of this phase is to sort the organ contours of the EquiVox case-base according to their similarity to $t$. As presented in 2.2, a classical algorithm for similarity calculation was used, namely the kNN algorithm. Considering $20 \mathrm{~cm} \leq$ human height $\leq 250 \mathrm{~cm}$, Equation 1 becomes:

$$
S_{i}=\frac{230-\left|h_{i}-h_{t}\right|}{230}
$$

\subsection{Adaptation of $3 D$ organ contours}

Once a matching case is retrieved, the expert can decide whether or not to use the organ representations of the most similar source cases, or to require the EquiVox platform to generate new ones, adapting the source cases to the 
target case. Indeed, assuming $S I M$ is the source case most similar to $t$, if $h_{S I M}$ and $h_{t}$ are too different, the expert may decide to adapt $S I M$ or even to create new organ contours which may be re-used for other problems later.

For all the solutions of the source cases, the same number of points defines the $3 \mathrm{D}$ contours of the lungs $\left(q_{\text {Lungs }}=26723\right)$, oesophagus $\left(q_{\text {oesophagus }}=\right.$ $7485)$, and heart $\left(q_{\text {Heart }}=7895\right)$. The points were plotted in the same order and in the same Cartesian coordinate system. Thus, the task of the organ contour-adaptation phase of EquiVox consists of interpolating the 3D coordinates of the points of $t$ in the same order and in the same Cartesian coordinate system. A Delaunay mesh can then be applied so as to create the contours of the organs of $t$. As concluded in [28], organ shapes and volumes of case $i$ depend on $h_{i}$ :

Dependency $\left(P_{O}^{i}, h_{i}\right)=T R U E \forall O \in\{$ Lungs, Heart,Oesophagus $\}$.

\subsubsection{Method}

Since the mesh and the number of points are not variable, the adaptation must be carried out on the point coordinates of the organ contours, point by point. Since no formal equation exists, we need a learning method to discover the rules that transform the coordinates of the points of one organ contour into other coordinates. Consequently, data-driven methods using inductive reasoning are the most suitable approaches; ANN and Fuzzy-ANN respond to these requirements. We chose ANN as the tool for this step, assuming this might serve as the basis for further work with Fuzzy-ANN if the first results were not convincing. We explored the possibility of using perceptrons with one or two hidden layers trained with a back propagation-based method, but other interpolation tools can easily be used by EquiVox: the purpose of this study was not to compare interpolation tools, but to provide and validate a general method for using one.

Each $C_{j}^{t, O}$ is interpolated from $C_{j}^{S I M, O}, h_{i}$ and $\Delta h=h_{S I M}-h_{t}$. Thus, there are $3 \mathrm{ANNs}$ (one for each organ) and Equation 2 becomes:

$$
P_{O}^{t}=\text { Interpolate }_{O}\left(\Delta h, h_{S I M}, P_{O}^{S I M}\right), O \in\{\text { Lungs, Heart, Oesophagus }\}
$$

ANN learning consists of determining the synaptic weights between neurons of different layers and also the optimum number of neurons in hidden layers. At first, a random number of neurons per and in the hidden layer(s), and random values for the synaptic weights are set. The synaptic weights are then computed until the mean distance $d_{w}$ between the interpolated and 
the expected phantoms is inferior to a given $\varepsilon$. After having optimised these synaptic weights, the mean distance $d_{n}$ between interpolated and expected yet unused phantoms (the ones in the validation set) is computed. If $d_{n}$ is superior to a given $\varepsilon$, the number of neurons in hidden layer(s) are changed and synaptic weights are computed one more time. This process is performed until $d_{n} \leq \varepsilon$.

Three ANNs use the same back-propagation L-BFGS training. The LBFGS method (Limited memory - BFGS) is an adaptation of the BFGS method (Broyden-Fletcher-Goldfarb-Shanno) which optimises the computational resources to use. The BFGS method is a quasi-Newton method, which approximates the value of the Hessian matrix of the second derivatives of the function to be minimised.

During the training phase of ANN, learning sets are generally divided into two parts: some of the elements are used to learn (optimisation of the synaptic weights) while others are used to validate (optimisation of the number of neurons in hidden layer(s)). In Figure 2, the 12 heights corresponding to $P_{1}$ to $P_{12}$ are reported on the same axis. Since we have a limited number of 3D Organ Contours (3DOC), we studied the interpolation accuracies regarding the composition of the learning and validation sets. We thus defined two main configurations and four possibilities for each as shown in Table 1. Generally, validation sets are composed of $10 \%$ of learning sets. For each possibility the validation set was therefore composed of one phantom: $P_{3}$ for Possibility $\sharp 1$, $P_{4}$ for Possibility $\sharp 2, P_{7}$ for Possibility $\sharp 3$ and $P_{9}$ for Possibility $\sharp 4$. A crossvalidation was then performed for the other sets. For the first configuration the constraint over the ANN input $\Delta h>0$ was added, whereas for the second one $\Delta h<0$ was required. Then, for each configuration, we explored the possibility of extracting one particular phantom of the learning set to include it in the validation set.

\section{Results}

The Equivox platform was implemented and tested on a Personal Computer equipped with an Intel Core i3 CPU, $2.53 \mathrm{GHz}$, and $4 \mathrm{GiB}$ RAM. Protégé was used to store the descriptor values. Two programming languages were used: Java and $\mathrm{C}++$. The retrieval phase, the GUIs, and the storage phase modules developed in Java call $\mathrm{C}++$ programs for the adaptation phase. All those programs were developed by our team. The phantoms were drawn using Rhino3D. The ANN learnings were performed in $\mathrm{C}++$ at 
the supercomputer facilities of the Mésocentre de calcul de Franche-Comté, containing 74 nodes based on Intel processors (4 to 6 cores) and 12 to 96 GB of ram. Each learning phase is mono-thread, so several learning phases could be simultaneously executed on a single node.

As explained above, for each of the three organs we tested two main configurations for EquiVox adaptation (respectively considering phantom heights inferior and superior to the target one) and four validation sets for each configuration. The detailed results for all three adapted organs are presented prior to presenting a global result analysis of the adaptation of 3DOC.

\subsection{Adaptation of lung contours}

Table 2 shows the distances obtained between interpolated and expected points of lungs (in $\times 10^{-3} \mathrm{~cm}$ ). Note that it is not possible to interpolate $P_{1}$ ' 3DOCs with the $(\Delta h>0)$ constraint and $P_{12}$ with the $(\Delta h<0)$ constraint.

With the constraint $\Delta h>0$, the learning configuration that gave the most accurate interpolations is the one with $\left\{P_{9}\right\}$ as the validation set. Most of the differences of approximately $0.02 \mathrm{~mm}$. The accuracies vary from 0.015 $\mathrm{mm}$ (interpolation of $P_{4}$ with $\left\{P_{9}\right\}$ as the validation set) to $0.642 \mathrm{~mm}$ (interpolation of $P_{5}$ with $\left\{P_{7}\right\}$ as the validation set). Nevertheless, even in such a case, the adaptation is sufficiently accurate since it is inferior to spatial resolutions of phantoms used by experts $(1.8 \mathrm{~mm} \times 1.8 \mathrm{~mm} \times 4.8 \mathrm{~mm})$. Interpolations with $\Delta h>0$ are generally better than with $\Delta h<0$. Contrary to what might be expected, the best adaptations of $P_{i+1}$ and $P_{i-1}$ are not always obtained when $\left\{P_{i}\right\}$ is the validation set. For example, the best interpolations of $P_{4}$ and $P_{2}$ are obtained when it is $P_{9}$ that is in the validation set, not $P_{3}$. Similarly, interpolations of $P_{8}$ are better with $\left\{P_{4}\right\}$ as the validation set than with $\left\{P_{9}\right\}$ and $\left\{P_{7}\right\}$. Thus, the best interpolations are not obtained when the 3D Lung contours of the phantom having the closest height are included in the validation set.

\subsection{Adaptation of heart contours}

The heart interpolations are globally more accurate than those for the lungs. As shown in Table 3, less important global deviation may be observed even if there is a great difference for the interpolation of $P_{5}$ with $\left\{P_{4}\right\}$ as the validation set $(0.646 \mathrm{~mm})$; all the other differences vary from $0.009 \mathrm{~mm}$ (interpolation of $P_{9}$ with $\left\{P_{7}\right\}$ as the validation set) to $0.098 \mathrm{~mm}$ (interpolation of $P_{3}$ with $\left\{P_{9}\right\}$ as the validation set). Unlike the lung interpolations, the configuration $\Delta h<0$ provides better interpolations, and globally, the 
configuration with $\left\{P_{3}\right\}$ as the validation set produced the most accurate interpolations of this organ's contours. As for the lungs, adaptations are accurate enough since they are inferior to spatial resolutions required by dosimetric reports, and additionally, no correlation is observed between the $3 \mathrm{DOC}$ used as the validation set and the best interpolated ones.

\subsection{Adaptation of oesophagus contours}

As shown in Table 4, the oesophagus interpolations were less accurate than the ones of the two previous organs. Specifically, they are roughly only one tenth as accurate as those for lungs: from $0.075 \mathrm{~mm}$ (interpolation of $P_{2}$ using $\left\{P_{4}\right\}$ as the validation set) to $14.3 \mathrm{~mm}$ (interpolation of $P_{5}$ using $\left\{P_{3}\right\}$ as the validation set). This is the only case for which the requirements of experts are not met. As for the other organs, no correlation can be found between the best interpolations of each 3DOC and the validation set used. Globally, the best interpolations for all 3 organs were obtained using $\left\{P_{3}\right\}$ as the validation set.

Finally, one configuration did not always provide the greatest accuracy for all the 3DOCs of one phantom. For example, considering $P_{11},\left\{P_{4}\right\}$ as the validation set and $\Delta h>0$ produced the best lung adaptations, whereas $\left\{P_{9}\right\}$ as the validation set and $\Delta h<0$ gave the best results for heart contours, while $\left\{P_{3}\right\}$ with $\Delta h<0$ was most accurate for the oesophagus. Similar results can be observed for all the other phantoms in addition to $P_{6}$. Indeed, the organs of $P_{6}$ are optimised with the same configuration $\left(\left\{P_{4}\right\}\right.$ and $\Delta h>$ $0)$.

\section{Discussion}

Figure 3 shows $P_{6}$ interpolated $3 \mathrm{DOC}$ and their accuracies using $\left\{P_{4}\right\}$ as the validation set and $\Delta h>0$. Each point is coloured according to its interpolation error, from blue (the lowest) to red (the highest). We can observe that there is no mesh problem and no artifact is added. In addition, $3 \mathrm{D}$ contours show a relatively realistic representation of lungs, heart and oesophagus.

As a general remark, we observe the greatest difficulty in the interpolation of $P_{5}$. However, in all but one of the tested cases, the requirements of the experts are satisfied. Indeed, only once are the interpolation deviations superior to the commonly used voxel dimensions used by radiation protection experts. In addition, the same configuration does not always give the best 
results. These observations and the deviations obtained may be explained by the fact that all the 3DOCs were designed manually and consequently contain biases.

These results emphasise the importance of the configuration and the 3DOC chosen for each set (validation and training), since the inclusion of one $3 \mathrm{DOC}$ in the validation set can generate accuracy which is twice as high, or greater than any another. In addition, the inclusion of one 3DOC in the validation set may introduce a bias for some interpolations while, at the same time, improving the accuracy of another target case. These biases may be eased by the capitalisation process: their impact will decrease as the number of source cases rises. Nevertheless, for each adaptation, choosing the training and validation sets very carefully can be another solution to alleviate the biases introduced by source case solutions.

In addition, the interpolations take only one to three seconds, a great help to physicians who usually need many hours to adapt one phantom. Hence, the generalisation of this method to the other organs may provide a tool that can be used in case of massive accidental exposure to radiation.

More generally, this paper presents a generic method for the use of interpolation tools to adapt a source case solution to a target case one in a CBR-system. We have proved the applicability of this generic method through its implementation in EquiVox, a CBR-HS which responds to the requirements of the particular issue of radiation protection. Indeed, the results tend to prove that the accuracy of adapted solutions mostly depends on the accuracy of the solutions of the source cases. Nevertheless, during its revision process, a CBR is able to evaluate (with the users help) the accuracy of adapted solutions. Indeed, in [13] A. Cordier et al. proposed to reuse in the adaptation phase the remarks made by users during revision processes. Future work will focus on how to take into account the revision remarks during CBR-adaptation phases driven by interpolation tools.

\section{Conclusion and Perspectives}

We have presented a generic method to use interpolation tools as influence functions for CBR-adaptation. This paper proves the applicability of this method since it was applied in EquiVox, a CBR-HS which can provide prototypes of 3D contours of organs based only on the subjects height. The resulting accuracies were analysed and discussed regarding the application 
domain requirements. These results confirm and quantify the general drawback of using interpolation as the means of adaptation in CBR systems [30]: imperfections are introduced into the adapted solutions. Consequently, two ways of improvement are now considered for EquiVox. The first consists in capitalising phantoms and 3DOC so as to progressively attenuate the imperfections of the solutions; the ANN interpolations, based on learning sets of greater and greater importance, will become better and better. Nevertheless, a second and more general option can be explored, which depends on associating vectors to the learning set so as to optimise interpolation accuracies and to determine, a priori, the best learning set/validation set for each target case.

Some of the interpolation errors were related to imperfections that may be found in the source case solutions. Thus, further investigation will need to focus on the elaboration of an adaptation algorithm capable of taking into account the reliability of a source case solution. Our goal, in other words, is to propose a tool that creates rules for the adaptation of target cases using confidence indices. This could be treated through the Genetic Algorithm and/or use of metaheuristics as Z. Liao et al. proposed in [31].

\section{Acknowledgements}

The authors wish to thank the Institut National de la Santé et de la Recherche Médicale, the Ligue Contre le Cancer, the Pays de Montbéliard Agglomération and the Société Française de Radioprotection for their financial aid, the National Institute of Radiological Protection and Nuclear Safety for their expertise, and John Olsen for the English review of this article.

\section{References}

[1] Kolodner, J.: Case-based reasoning. San Mateo, CA: Morgan Kaufmann (1993)

[2] Melis, E., Lieber, J., Napoli, A.: Reformulation in Case-Based Reasonning. In:Forth European Workshop on Case-Based Reasoning, Lecture Notes in Artificial Intelligence, Springer, 1488:172-183 (1998)

[3] Fuchs, B., Lieber, J., Mille, A., Napoli, A.: A general strategy for adaptation in Case-Based Reasoning. In: LIRIS Report RR-LIRIS-2006-016, http://liris.cnrs.fr/publis/?id=2477 (2006) 
[4] Lieber, J.: Application of the Revision Theory to Adaptation in CaseBased Reasonning: the Conservative Adaptation. In: Proceedings of the 7th International Conference on Case-Based Reasonning, Lecture Notes in Artificial Intelligence, Springer, 4626:239-253 (2007)

[5] Bichindaritz, I., Marling, C.: Case-based reasoning in the health sciences: Whats next?. In: Artificial Intelligence in Medicine 36: 127-135 (2006)

[6] Bichindaritz, I.: Prototypical Cases for Knowledge Maintenance in Biomedical CBR. In: Proceedings of International Conference on CaseBased Reasoning, Belfast, 492-506 (2007)

[7] Little, S., Colatino, S., Salvetti, O., Perner, P.: Can Prototype-Based Classifica-tion be a good Method for Biomedical Applications?. In: Trans. MLDM (MLDM) 2(1): 44-61 (2009)

[8] DAquin, M., Lieber, J., Napoli, A.: Adaptation Knowledge Acquisition: A Case Study for Case-Based Decision Support in Oncology. In: Computational Intelligence, 161-176 (2006)

[9] Babita Pandey, Michra, R.B.: An integrated intelligent computing model for the interpolation of EMG based neuromuscular disease. In: Expert Systems With Applications 36, pp. 9201-9213 (2009)

[10] Cover, T., Hart, P.: Nearer Neighbour Pattern Classification. In: IEEE Transactions on Information Theory 13:21-27 (1967)

[11] Knight, B., Woon, F.L.: Case Based Adaptation Using Interpolation over Nominal Values. In: Research and development in Intelligent Systems, Springer, 21:73-86 (2005)

[12] Shepard, D.: A Two-Dimensional interpolation Function for irregularity Spaced Data. In: Proceedings of the 23rd National Conference, ACM, 517-523 (1968)

[13] Cordier, A., Fuchs, B., Mille, A.: Engineering and learning of adaptation knowledge and Case-Based Reasoning. In: Proceedings of the 15th International Conference on Knowledge Engineering and Knowledge Management - EAKW 2006, Springer-Verlag, 303-317 (2006) 
[14] Broggio, D. and Zhang, B. and de Carlan, L. and Desbrée, A. and Lamart, S. and le Guen, B. and Bailloeuil, C. and Franck, D.: Analytical and Monte Carlo assessment of activity and local dose after a wound contamination by activation products. In: Health Physics 96(2):155-163 (2009)

[15] Huet, C. and Lemosquet, A. and Clairand, I. and Rioual, J.B. and Franck, D. and de Carlan, L. and Aubineau-Lanièce, I. and BottollierDepois, J.F.: SESAME: a software tool for the numerical dosimetric reconstruction of radiological accidents involving external sources and its application to the accident in Chile in December 2005. In: Health Physics 96(1):76-83 (2009)

[16] Bichindaritz, I.: Case-based reasoning in the health sciences: Why it matters for the health sciences and for CBR. In: Althof, K.-D.; Bergmann, R.; Minor, M.; and Hanft, A., eds., Advances in Case-Based Reasoning: 9th European Conference, ECCBR, 1-17. Berlin: Springer (2008)

[17] Bichindaritz, I.: Advances in case-based reasoning in the health sciences. In: Artificial Intelligence in Medicine 51: 75-79 (2011)

[18] Diaz, F., Fdze-Riverola, F., Corchado, J.M.: Gene-CBR: A Case-Based Reason-ing Tool for Cancer Diagnosis using Microarray Datasets. In: Computational Intelligence, 254-258 (2006)

[19] El Balaa, Z., Strauss, A., Uziel, P., Maximini, K., Traphoner, R.: FMUltranet: A Decision Support System Using Case-Based Reasoning Applyied to Ultrasono-graphy. In: McGinty, L. (ed.) Workshop Proceedings of the Fifth International Conference on Case-Based Reasoning, NTNU, Trondheim, Norway, 37-44 (2003)

[20] Monati, S.: Case-Based Reasoning for Managing Non-Compliance with Clinical Guidelines. In: Wilson, D.C., Khemani, D. (ed.) Proceedings of Case-Based Rea-soning in Health Science Workshop, ICCBR, Belfast, 325-336 (2007)

[21] Reategui, E.B., Campbell, J.A., Leaob, B.F.: Combining a neural network with case-based reasoning in a diagnostic system. In: Artificial Intelligence in Medicine 9:5-27 (1997) 
[22] Henriet, J., Leni, P.E., Laurent, R., Roxin, A., Chebel-Morello, B., Salomon, M., Farah, J., Broggio, D., Franck, D., Makovicka, L.: Adapting numerical representations of lung contours using Case-Based Reasoning and Artificial Neural Networks. In: B. Daz Agudo and I. Watson (Eds.): ICCBR 2012, Artificial Intelligence series, Springer, Heidelberg, LNCS 7466, pp. 137-151 (2012)

[23] McCulloch, W.S. and Pitts, W.: A logical calculus of the ideas immanent in nervous activity. In: Bulletin of mathematical biology, Springer, 5(4):115-133 (1943)

[24] Valentin, J.: Basic anatomical and physiological data for use in radiological protection: reference values:: ICRP Publication 89. In: Annals of the ICRP, Elsevier, 32(3-4):1-277 (2002)

[25] Farah, J. and Broggio, D. and Franck, D.: Examples of Mesh and NURBS modeling for in vivo lung counting studies. In: Radiation Protection Dosimetry, NTP, 144(1-4):344-348 (2011)

[26] Broggio, D., Beurrier, J., Bremaud, M., Desbrée, A., Farah, J., Huet, C., Franck, D.: Construction of an extended library of adult male 3D models: rationale and results. in: Phys. Med. Biol. 56: 76597692 (2011)

[27] McNeel, R. (2003) Modeling tools for designers. Rhinoceros Web Site, http://www.rhino3d.com

[28] Clairand, I. and Bouchet, L.G. and Ricard, M. and Durigon, M. and Di Paola, M. and Aubert, B.: Improvement of internal dose calculations using mathematical models of different adult heights. In: Physics in medicine and biology, IOP Publishing, 45:2771-2785 (2000)

[29] Christensen, G.E.: Deformable shape models for anatomy. Thesis, Washington University (1994)

[30] Chatterjee, N. and Campbell, J.A.: Interpolation as a means of fast adaptation in case-based problem solving. In: Proceedings Fifth German Workshop on Case-Based Reasoning 65-74 (1997)

[31] Zhenliang Liao, Xuewei Maoa, Phillip M. Hannam, Tingting Zhao: Adaptation methodology of CBR for environmental emergency preparedness system based on an Improved Genetic Algorithm. In: Expert Systems With Applications 39:7029-7040 (2012) 


\section{Figures}

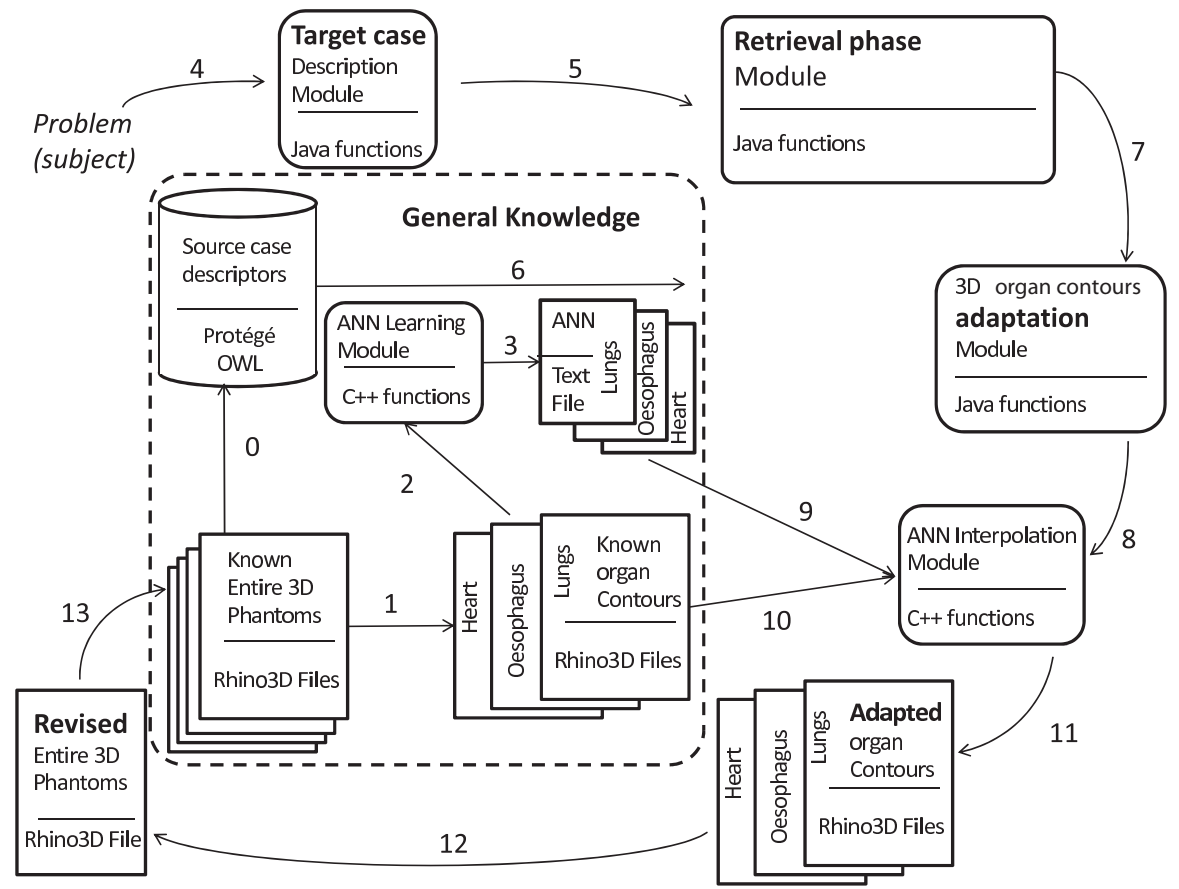

Figure 1: Data flows over the EquiVox architecture.

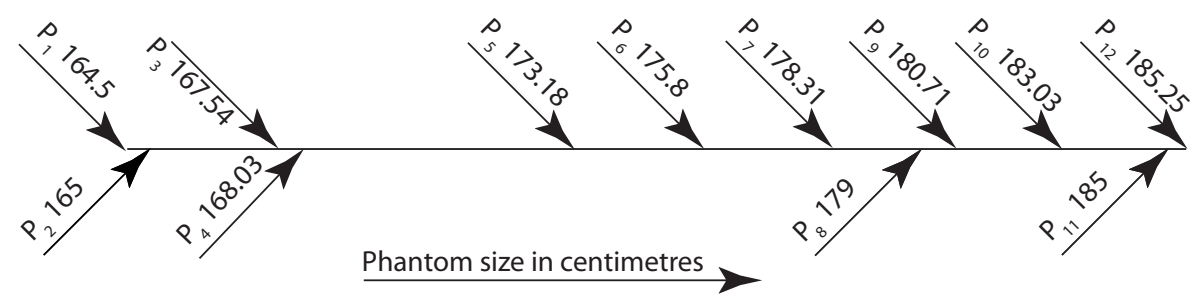

Figure 2: Phantom heights of the available 3D organ contours. 


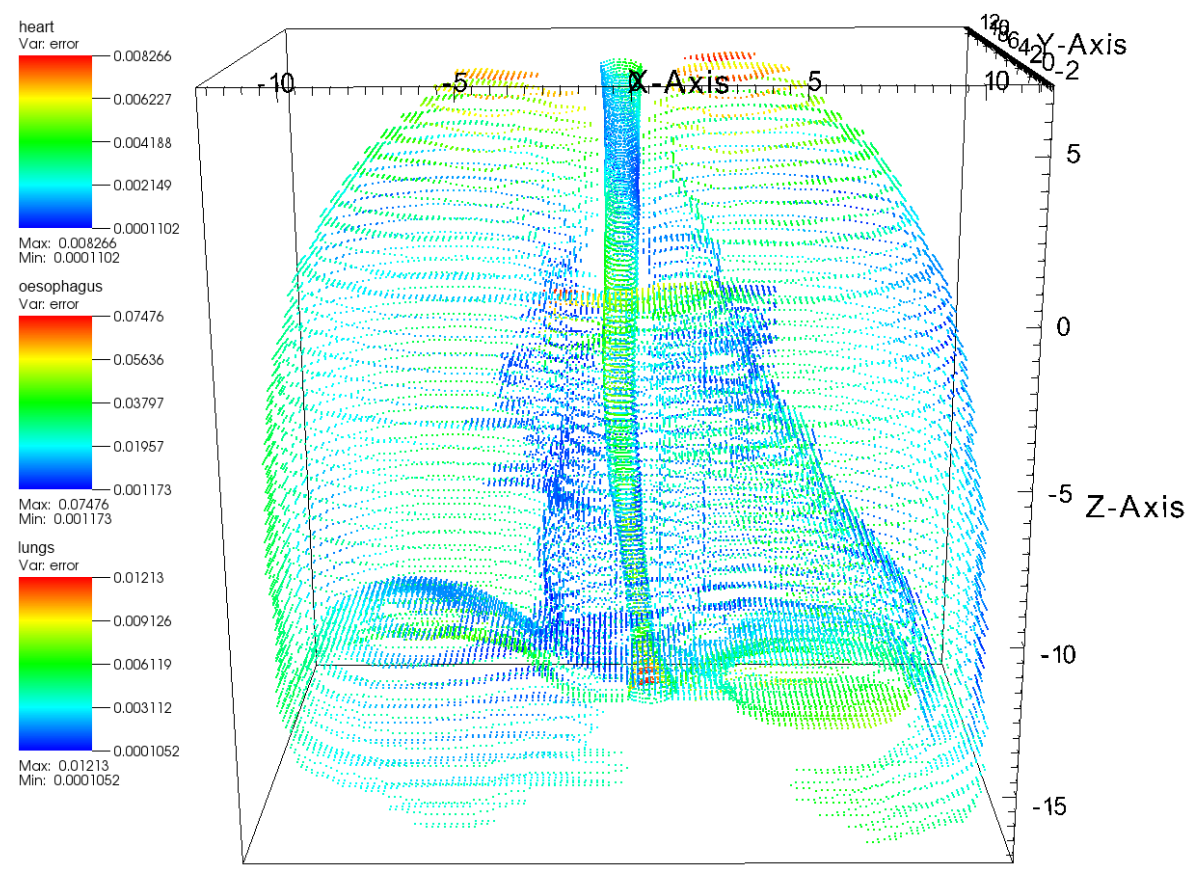

Figure 3: Representation of $P_{6}$ interpolated organs with $\left\{P_{4}\right\}$ as the validation set and $\Delta h>0$. 


\section{Tables}

\begin{tabular}{lccc}
\hline & Test set & Validation set & Learning set \\
\hline Possibility $\sharp 1$ & $\left\{P_{i}\right\}, i \in\{1 . .12\} i \neq 3$ & $\left\{P_{3}\right\}$ & $\bigcup_{j \in\{1.12\}, j \neq 3, j \neq i}\left\{P_{j}\right\}$ \\
Possibility $\sharp 2$ & $\left\{P_{i}\right\}, i \in\{1 . .12\} i \neq 4$ & $\left\{P_{4}\right\}$ & $\bigcup_{j \in\{1 . .12\}, j \neq 4, j \neq i}\left\{P_{j}\right\}$ \\
Possibility $\sharp 3$ & $\left\{P_{i}\right\}, i \in\{1 . .12\} i \neq 7$ & $\left\{P_{7}\right\}$ & $\bigcup_{j \in\{1.12\}, j \neq 7, j \neq i}\left\{P_{j}\right\}$ \\
Possibility $\sharp 4$ & $\left\{P_{i}\right\}, i \in\{1 . .12\} i \neq 9$ & $\left\{P_{9}\right\}$ & $\bigcup_{j \in\{1 . .12\}, j \neq 9, j \neq i}\left\{P_{j}\right\}$ \\
\hline
\end{tabular}

Table 1: Learning, validation and test sets tested. 


\begin{tabular}{cccccccccccccc}
\hline Validation & constraint & \multicolumn{11}{c}{ Tested 3DOC } \\
set & $\Delta h$ & $P_{1}$ & $P_{2}$ & $P_{3}$ & $P_{4}$ & $P_{5}$ & $P_{6}$ & $P_{7}$ & $P_{8}$ & $P_{9}$ & $P_{10}$ & $P_{11}$ & $P_{12}$ \\
\hline$P_{3}$ & $>0$ & $/$ & 3.5 & $/$ & 8.7 & 23.9 & 6.7 & 2.7 & 4.1 & 17.5 & 25.5 & 3.7 & 8.9 \\
$P_{3}$ & $<0$ & 2.6 & 4.1 & $/$ & 13.4 & 4.3 & 6.9 & 7.9 & 8.9 & 24.9 & 20.2 & 7.5 & $/$ \\
\hline$P_{4}$ & $>0$ & $/$ & 6.2 & 42.9 & $/$ & 38.7 & 3.5 & 2.1 & 2.0 & 1.9 & 31.7 & 1.9 & 8.5 \\
$P_{4}$ & $<0$ & 1.7 & 2.8 & 3.7 & $/$ & 4.5 & 6.4 & 6.2 & 6.2 & 4.3 & 11.8 & 7.5 & $/$ \\
\hline$P_{7}$ & $>0$ & $/$ & 4.2 & 16.4 & 3.3 & 64.2 & 6.3 & $/$ & 7.4 & 2.2 & 4.9 & 2.9 & 2.3 \\
$P_{7}$ & $<0$ & 5.7 & 2.8 & 2.7 & 45.5 & 3.4 & 7.4 & $/$ & 8.9 & 5.0 & 15.4 & 4.9 & $/$ \\
\hline$P_{9}$ & $>0$ & $/$ & 2.4 & 3.4 & 1.5 & 12.0 & 6.8 & 2.9 & 6.2 & $/$ & 8.3 & 3.3 & 4.4 \\
$P_{9}$ & $<0$ & 3.6 & 7.9 & 3.2 & 4.4 & 6.6 & 5.7 & 11.1 & 4.1 & $/$ & 9.1 & 7.3 & $/$ \\
\hline
\end{tabular}

Table 2: Distances $\left(\times 10^{-3} \mathrm{~cm}\right)$ between interpolated and expected points of lung contours.

\begin{tabular}{cccccccccccccc}
\hline Validation & constraint & \multicolumn{10}{c}{ Tested 3DOC } \\
set & $\Delta h$ & $P_{1}$ & $P_{2}$ & $P_{3}$ & $P_{4}$ & $P_{5}$ & $P_{6}$ & $P_{7}$ & $P_{8}$ & $P_{9}$ & $P_{10}$ & $P_{11}$ & $P_{12}$ \\
\hline$P_{3}$ & $>0$ & $/$ & 3.1 & $/$ & 4.9 & 11.1 & 4.2 & 1.3 & 7.6 & 15.3 & 13.2 & 2.2 & 3.7 \\
$P_{3}$ & $<0$ & 2.5 & 2.3 & $/$ & 4.9 & 2.4 & 4.1 & 4.3 & 5.4 & 2.4 & 4.6 & 3.6 & $/$ \\
\hline$P_{4}$ & $>0$ & $/$ & 3.5 & 24.0 & $/$ & 64.6 & 2.3 & 1.9 & 2.1 & 1.6 & 1.9 & 2.3 & 3.9 \\
$P_{4}$ & $<0$ & 1.2 & 2.6 & 2.4 & $/$ & 6.1 & 4.6 & 3.5 & 11.5 & 2.0 & 3.3 & 1.3 & $/$ \\
\hline$P_{7}$ & $>0$ & $/$ & 2.4 & 8.6 & 9.7 & 18.7 & 4.5 & $/$ & 3.9 & 0.9 & 2.9 & 2.4 & 1.5 \\
$P_{7}$ & $<0$ & 1.4 & 8.2 & 0.9 & 6.6 & 1.5 & 4.3 & $/$ & 2.3 & 11.4 & 1.9 & 1.2 & $/$ \\
\hline$P_{9}$ & $>0$ & $/$ & 1.2 & 9.8 & 1.2 & 15.3 & 4.0 & 1.3 & 4.3 & $/$ & 5.7 & 1.8 & 1.8 \\
$P_{9}$ & $<0$ & 1.1 & 1.3 & 2.5 & 2.4 & 3.9 & 2.8 & 5.5 & 0.8 & $/$ & 26.0 & 1.1 & $/$ \\
\hline
\end{tabular}

Table 3: Distances $\left(\times 10^{-3} \mathrm{~cm}\right)$ between interpolated and expected points of heart contours. 


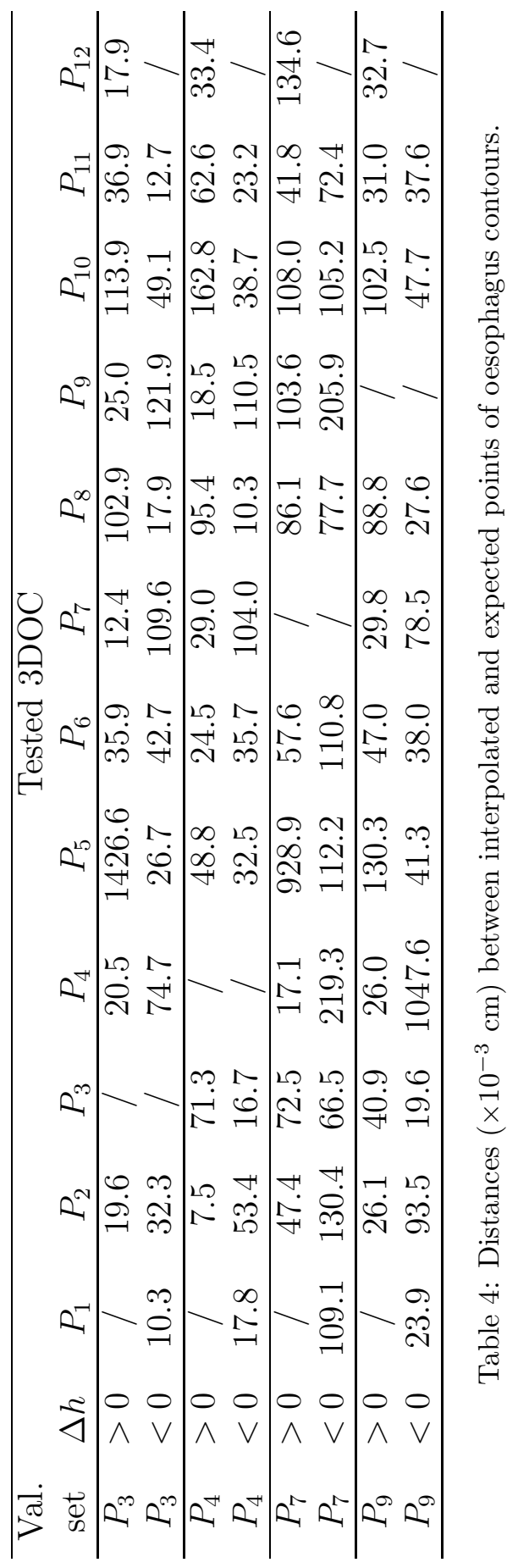

